\title{
Extracorporeal Shock Wave Therapy for the Treatment of Non-Union of a Canine Mandibular Fracture
}

\author{
Olivia J. Oginska ${ }^{1}$ Richard Whitelock ${ }^{1}$ Kirsten Hausler ${ }^{2}$ Amy Stelman ${ }^{1}$ Matthew J. Allen ${ }^{1}$ \\ ${ }^{1}$ Department of Veterinary Medicine, Cambridge University, \\ Cambridge, United Kingdom of Great Britain and Northern Ireland \\ 2 Zentum für Tierphysiotherapie Dr. Kirsten Häusler, Stuttgart, Germany \\ Address for correspondence Olivia J. Oginska, DVM, MRCVS, \\ Department of Veterinary Medicine, Cambridge University, Madingley \\ Road, Cambridge CB3 0ES, United Kingdom of Great Britain and \\ Northern Ireland (e-mail: oginskaolivia@gmail.com). \\ VCOT Open 2019;2:e56-e59.
}
Abstract
Keywords
- fracture healing
- extracorporeal shock wave
- non-union
- mandible
- dog

The use of extracorporeal shock wave therapy (ESWT) for the treatment of delayed or non-union of long bone fractures has been described in human patients with encouraging results. This case report describes the successful treatment of a mandibular fracture non-union with ESWT. To authors' knowledge, this is the first report of the clinical use of ESWT for the management of a non-union in a dog.

\section{Introduction}

Since its introduction in the 1980 s as a non-invasive treatment for urolithiasis, extracorporeal shock wave therapy (ESWT) has become an increasingly popular treatment option for musculoskeletal injuries in human and veterinary medicine. ${ }^{1-6}$ Extracorporeal shock wave therapy involves delivery of a sonic pulse (acoustic pressure or sound wave) to target tissues below the skin. The sonic pulse is characterized by a rapid increase in pressure, a high peak pressure that decays rapidly, a short life cycle and a broad frequency spectrum. ${ }^{7,8}$ Shock waves have been used clinically in both human and veterinary medicine, mainly to treat soft tissue disease such as tendinopathy or plantar fasciitis. ${ }^{7}$ Extracorporeal shock wave therapy has also attracted attention as a non-invasive approach for stimulating fracture healing. Thirty years ago, it was reported that shock waves cause micro-fractures and induce new bone formation in the rabbit femur. ${ }^{1}$ Further studies have proven that shock waves cause periosteal detachment and micro-fractures on the endosteal surface of the canine femoral cortex, followed 2 months later by callus formation beneath the detached periosteum. ${ }^{2}$ Another study documented enhanced callus formation 12 weeks after a single session of ESWT applied over $3 \mathrm{~mm}$ segmental defects in adult dogs. ${ }^{3}$ Based on these preclinical studies, ESWT was translated into the human orthopaedic clinic, producing encouraging results in patients with nonunions of long bone fractures. ${ }^{4,5,9,10}$ Less is known about the effects of ESWT on fracture healing in veterinary patients, and although there has been an isolated report on the use of ESWT to stimulate bone healing following tibial-plateau-levelling osteotomy, ${ }^{5}$ to our knowledge there has not been a clinical report on the use of ESWT for managing a non-union in a dog.

\section{Case Description}

A 4-year-old male neutered Chihuahua was referred to the Queen's Veterinary School Hospital in Cambridge 7 days after being diagnosed with a fracture in the caudal third of the body of the left mandible ( $\mathbf{- F i g}$. 1). The dog had initially been managed conservatively with pain relief (meloxicam $3 \mathrm{~kg}$ dose PO) and a soft diet, but his appetite diminished over the following week, prompting referral to our hospital. On clinical examination, the left lower carnassial (309) was identified as being fractured at the level of the mandibular fracture. The mandibular fracture was extremely unstable, causing significant pain on manipulation. The dog was prepared for surgery with the owner's consent; tooth extraction and surgery were performed under general anaesthesia. For premedication, the dog was administered medetomidine $(2 \mu \mathrm{g} / \mathrm{kg}$ intravenous [IV]) and methadone ( $0.2 \mathrm{mg} / \mathrm{kg}$ IV). Anaesthesia was induced
License terms Stuttgart · New York
DOI https://doi.org/

$10.1055 / \mathrm{s}-0039-3400617$. ISSN $2625-2325$.
May 20, 2019

accepted after revision

September 4, 2019 


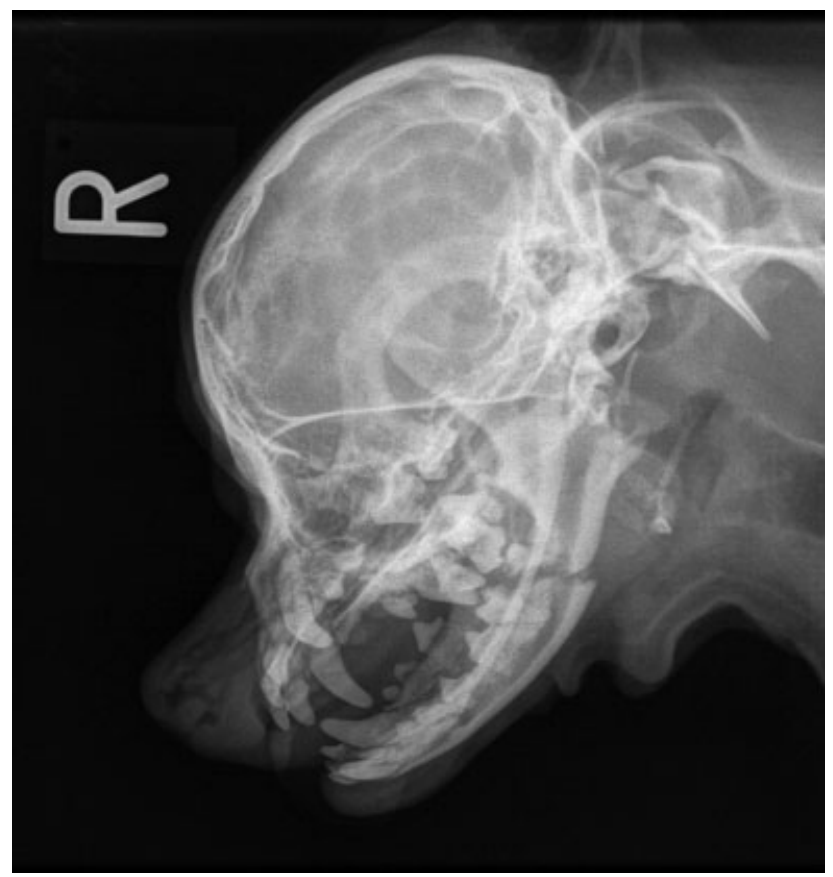

Fig. 1 Preoperative lateral oblique radiograph of the patient's left mandible showing a fracture in the caudal third of the body of the mandible.

with propofol ( $1 \mathrm{mg} / \mathrm{kg}$ IV to effect) and maintained with isoflurane and oxygen. Fentanyl in constant rate infusion $(8 \mu \mathrm{g} / \mathrm{kg} / \mathrm{min})$ proceeded by a loading dose $(0.5 \mu \mathrm{g} / \mathrm{kg}$ IV) was used for the intraoperative analgesia as an addition to the mandibular nerve block (bupivacaine $1 \mathrm{mg} / \mathrm{kg}$ ). The broken carnassial was removed and the fracture stabilized with a type $1 \mathrm{~A}$ external skeletal fixator using four $1.6 \mathrm{~mm}$ Ellis pins (Veterinary Instrumentation Ltd., Sheffield, UK) bonded to a connecting bar with polymethylmethacrylate bumper (Technovit 6091; Kulzer GmbH, Wehrheim, DE). The most rostral pin was placed between the roots of the left lower second premolar (306) and the left lower third premolar (307), whereas the second most rostral pin placed rostral to the roots of the left lower carnassial (309) that was removed at the beginning of the procedure. The third and fourth pins were placed in the ramus of the mandible. Good fracture alignment and bone apposition were achieved (-Fig. 2). The patient was reevaluated 3 weeks after the surgery and no signs of any complication were detected. All pin sites were clean and dry. Follow-up radiographs were obtained 8 weeks after application of the external fixator ( 9 weeks after the initial injury) and these revealed incomplete bone healing with only modest evidence of active new bone formation ( - Fig. 3). As before, all pin sites were clean and dry, and the fixator was stable on palpation. A diagnosis of a non-union of the mandibular fracture was made. The same day the patient was heavily sedated (methadone $0.2 \mathrm{mg} / \mathrm{kg}$ plus medetomidine $0.02 \mathrm{mg}$ / $\mathrm{kg}$ IV) and treated with focal shockwave (Duolith SD1 Ultra, Storz Medical AG; Tägerwilen, Switzerland). Based on prior clinical experience with using shockwave for applications in humans and dogs, the machine was set to deliver 750 pulses at an energy density of $0.15 \mathrm{~mJ} / \mathrm{cm}^{2} .{ }^{10,11}$ The probe was applied direct to the skin over the ventral mandible, with an interme-

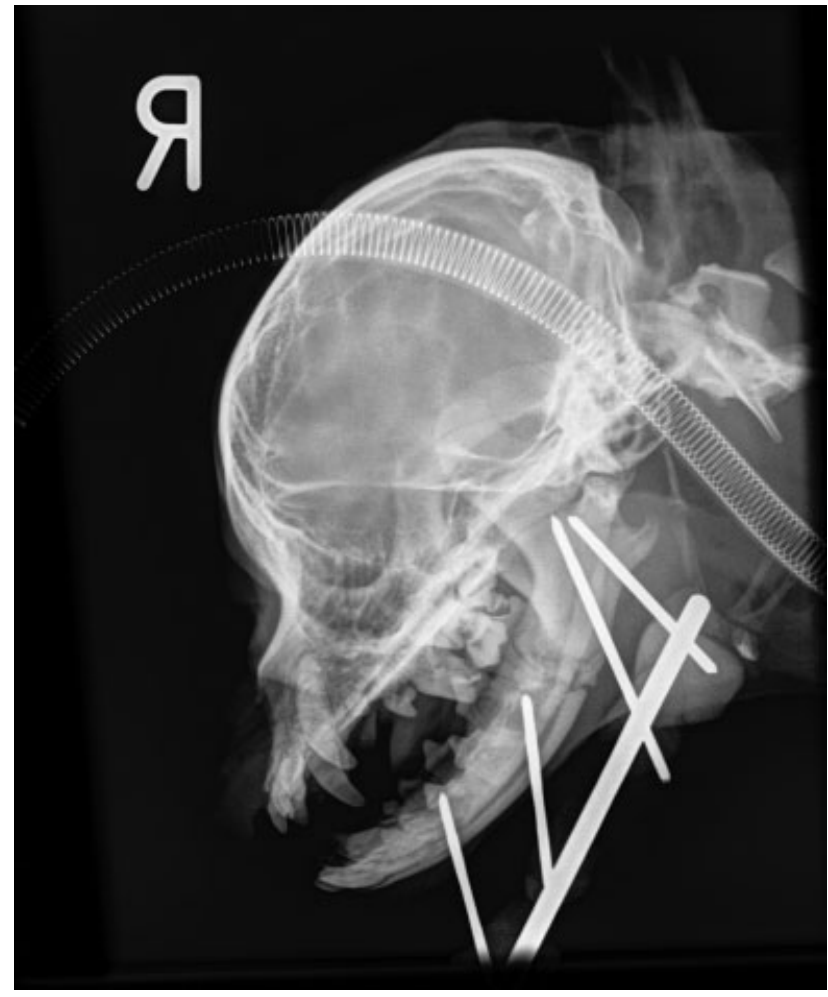

Fig. 2 Postoperative lateral oblique radiograph of the patient's left mandible showing good fracture alignment and bone apposition.

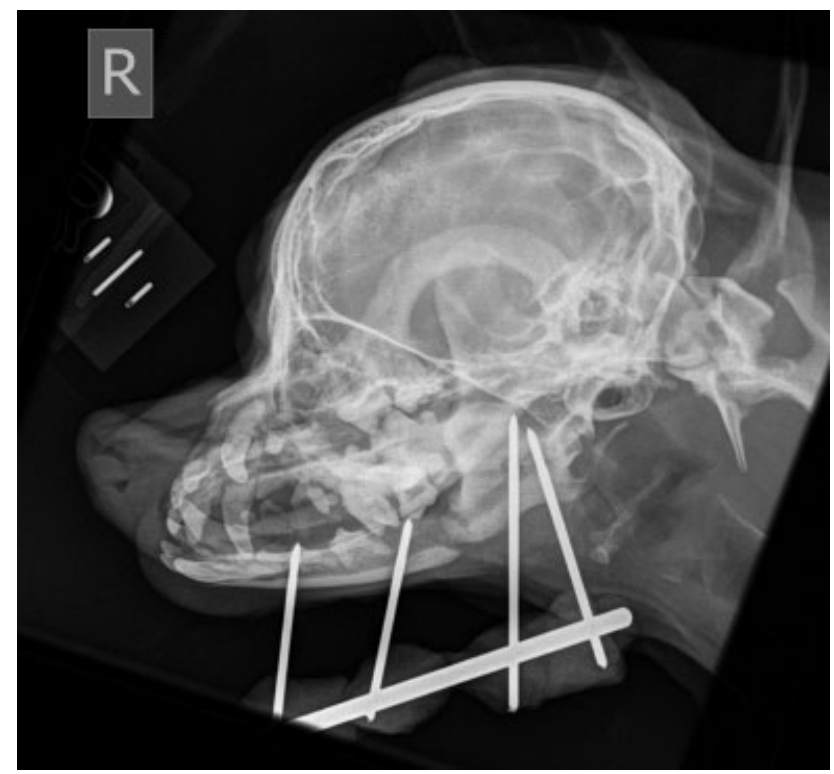

Fig. 3 Lateral oblique radiograph of the patient's left mandible (8 weeks after closed stabilization of the mandibular fracture with an external skeletal fixator) showing absence of bone healing.

diate layer of gel as an acoustic coupler. The probe was angled dorsally and medially, directly over the fracture site ( - Fig. 4). Additional treatments were delivered 2 and 4 weeks later, using the same instrument settings. No other treatment or medication was given. Radiographs were repeated 6 weeks after the first application of shockwave treatment and showed good bone healing with robust callus formation across the 


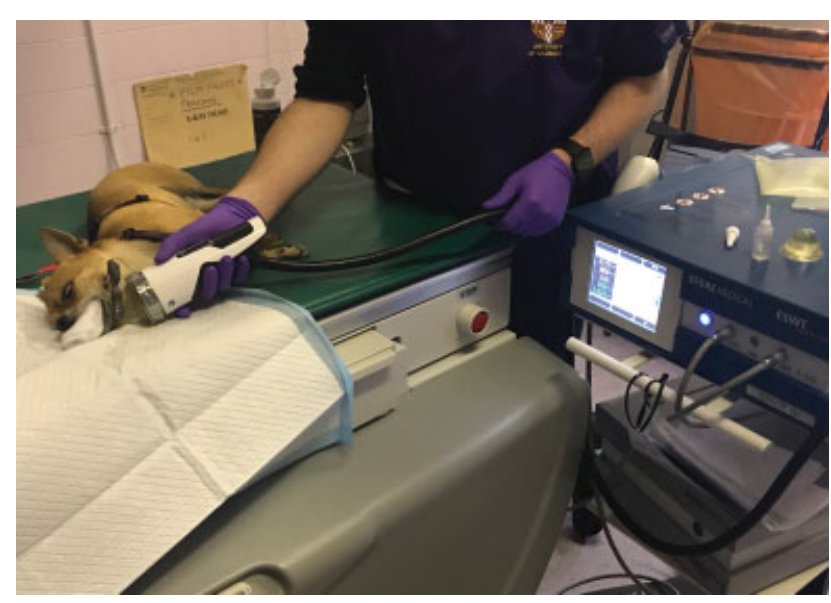

Fig. 4 Patient undergoing extracorporeal shock wave therapy treatment.



Fig. 5 Lateral oblique radiograph of the patient's left mandible (taken 6 weeks after the first post-surgical imaging and after three extracorporeal shock wave therapy treatments) showing good bone healing with robust callus formation across the original fracture plane.

original fracture plane (-Fig. 5). The external skeletal fixator was then removed as the mandible was stable on manipulation. The patient was discharged the same day. The owner was contacted by phone 4 months after the final session of ESWT to gauge clinical progress; the dog had not experienced any longterm complications and was able to eat and drink without any problems. The owner reports the dog as having a normal quality of life following successful healing of this fracture.

\section{Discussion}

The incidence of delayed and non-union fractures in dogs is unknown, although it appears to be higher in small breeds. ${ }^{12}$ Certain fracture sites are more prone to the development of fracture non-unions, with common examples being the distal radius and ulna in small and toy breeds. ${ }^{8,11}$ Mandibular fractures, which account for 1.5 to $3 \%$ of all fractures in dogs, also appear to be at risk of delayed or non-union, with complications rates following surgical management as high as $34 \%{ }^{13}$ The reasons for the high complication rate are believed to include impaired blood supply, instability in comminuted fractures and delays in treatment. ${ }^{9,14}$ The latter could have been one of the factors that contributed to non-union in the patient described in this case report, as he was referred for the surgical stabilization a week after the accident. Osteomyelitis, secondary to periodontal disease, and the presence of a tooth in the fracture line have also been reported as adversely affecting normal fracture healing. ${ }^{9,14}$ The latter was a factor in this clinical case since there was a broken carnassial tooth associated with the mandibular fracture plane. The radiographic signs of a non-union include the presence of minimal evidence of a reparative bone response but continued presence of a radiolucent line through the fracture site at a time when one would normally expect bone healing. ${ }^{12}$ These features were evident on the radiographs of our patient 8 weeks after the surgical stabilization ( 9 weeks after the original injury).

Treatment of a delayed union or non-union focuses on improving the local physiological and mechanical environment to encourage healing to proceed. The use of bone grafts, surgical debridement of the fracture bone ends, additional stabilization, fracture compression and biophysical stimulation with electrical/electromagnetic stimulation and pulsed ultrasound have all been described in humans and, to more variable levels, in dogs. ${ }^{8,12,15}$ The high costs and morbidity of surgical revision highlight the importance of non-surgical treatment options. In this patient, ESWT was selected because it is affordable, non-invasive and of proven efficacy in humans.

The precise mechanism underlying the therapeutic effects of ESWT remains poorly defined but it appears to involve a combination of micro-fracture formation, enhanced neovascularization and osteoblastic activity, increased levels of nitric oxide, activation of osteogenic growth factors and enhanced osteogenic differentiation of mesenchymal stem cells. All of those mechanisms can facilitate bone healing in the case of delayed or non-union, where cellular proliferation and new bone formation have ceased..$^{5,6,15}$ It is recognized that bone cell stimulation depends on the total amount of energy applied, rather than on single physical parameter. ${ }^{7}$

In humans, ESWT has been reported to be extremely successful in treating non-unions in long bone, with $79 \%$ of cases going on to osseous union. ${ }^{15}$ Although ESWT has been proven to encourage bone healing in research dogs with experimental non-unions, ${ }^{10}$ as well as in clinical patients undergoing tibial-plateau-leveling osteotomy, ${ }^{5}$ to date there have not been any reports on the use of ESWT to promote the healing of a delayed or non-union in a clinical case. Our patient underwent three consecutive sessions, at 2-week intervals, but this may not be the optimal treatment protocol. We selected our approach on the basis of the available literature, the patient's physical condition, the owner's financial resources and potential concerns over the client's compliance were the dog to undergo additional surgery. 
In conclusion, three doses of non-invasive ESWT stimulated bone healing in a dog with a non-union of a mandibular fracture. Although this is an isolated case report, without an appropriate control for comparison, the use of ESWT was the only change made in the management of the case and a robust callus developed at the fracture site 6 weeks of the first treatment. There was no evidence of short- or long-term complications with the use of ESWT.

\section{Author Contribution}

Clinical care of the patient was undertaken by Richard Whitelock and Olivia Oginska. Shockwave therapy was performed by Matthew Allen, Amy Stelman and Kirsten Hausler. The manuscript was written by Olivia Oginska and Matthew Allen. All of the authors reviewed and approved the final manuscript.

\section{Conflict of Interest}

Authors have received in-kind support from Storz Medical.

\section{References}

1 Ikeda K, Tomita K, Takayama K. Extracorporeal shock wave induced bone formation. Iyodenshi To Seitai Kogaku 1993; $31: 28$

2 Ikeda K, Tomita K, Takayama K. Application of extracorporeal shock wave on bone: preliminary report. J Trauma 1999;47(05): 946-950

3 Wang CJ, Huang HY, Chen HH, Pai CH, Yang KD. Effect of shock wave therapy on acute fractures of the tibia: a study in a dog model. Clin Orthop Relat Res 2001;(387):112-118
4 Ikeda K. [Bone fracture and the healing mechanisms. Application of the extracorporeal shock wave on treatment of fracture]. Clin Calcium 2009;19(05):718-725

5 Kieves NR, MacKay CS, Adducci K, et al. High energy focused shock wave therapy accelerates bone healing. A blinded, prospective, randomized canine clinical trial. Vet Comp Orthop Traumatol 2015;28(06):425-432

6 Wang CJ, Huang HY, Pai CH. Shock wave-enhanced neovascularization at the tendon-bone junction: an experiment in dogs. J Foot Ankle Surg 2002;41(01):16-22

7 Zelle BA, Gollwitzer H, Zlowodzki M, Bühren V. Extracorporeal shock wave therapy: current evidence. J Orthop Trauma 2010;24 (Suppl 1):S66-S70

8 Jackson LC, Pacchiana PD. Common complications of fracture repair. Clin Tech Small Anim Pract 2004;19(03):168-179

9 Mathog RH, Toma V, Clayman L, Wolf S. Nonunion of the mandible: an analysis of contributing factors. JOral Maxillofac Surg 2000;58(07):746-752, discussion 752-753

10 Johannes EJ, Kaulesar Sukul DM, Matura E. High-energy shock waves for the treatment of nonunions: an experiment on dogs. J Surg Res 1994;57(02):246-252

11 Leal C, D'Agostino C, Gomez Garcia S, Fernandez A. Current concepts of shockwave therapy in stress fractures. Int J Surg 2015;24(Pt B):195-200

12 Nunamaker D, Rhinelander F, Heppenstall R. 2019Delayed Union, Nonunion, and Malunion (1-Jan-1985). Available at: https:// www.researchgate.net/publication/266495340_Delayed_Union_ Nonunion_and_Malunion_1-Jan-1985

13 Harasen G. Maxillary and mandibular fractures. Can Vet J 2008;49 (08):819-820

14 Bruno JR, Kempers KG, Silverstein K. Treatment of traumatic mandibular nonunion. JCraniomaxillofac Trauma 1999;5(02):27-32

15 Wang CJ, Yang KD, Ko JY, Huang CC, Huang HY, Wang FS. The effects of shockwave on bone healing and systemic concentrations of nitric oxide (NO), TGF-beta1, VEGF and BMP-2 in long bone non-unions. Nitric Oxide 2009;20(04):298-303 